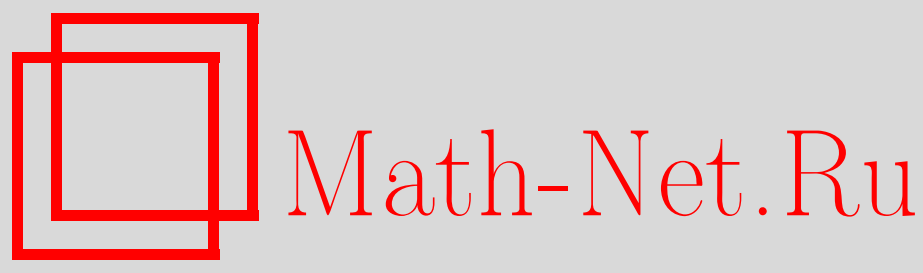

П. А. Вшивцева, В. И. Денисов, И. П. Денисова, Интегральное соотношение для тензорных полиномов, ТМФ, 2011, том 166, номер 2, 216-224

DOI: https://doi.org/10.4213/tmf6604

Использование Общероссийского математического портала Math-Net.Ru подразумевает, что вы прочитали и согласны с пользовательским соглашением http://www . mathnet.ru/rus/agreement

Параметры загрузки:

IP: 52.90 .164 .192

26 апреля 2023 г., 10:58:08

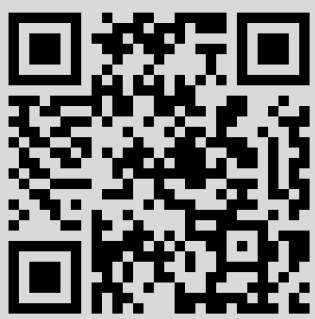




\title{
ФИЗИКА
}

Том 166, № 2

февраль, 2011

(C) 2011 г. П. А. Вшивцева*, В. И. Денисов* , И. П. Денисова*

\section{ИНТЕГРАЛЬНОЕ СООТНОШЕНИЕ ДЛЯ ТЕНЗОРНЫХ ПОЛИНОМОВ}

\begin{abstract}
Доказаны две леммы и одна теорема, позволяющие проводить интегрирование произведения произвольного числа единичных векторов на полиномы Лежандра по поверхности сферы произвольного радиуса. Такие интегральные тензорные выражения возникают при решении неоднородных уравнений Гельмгольца, правая часть которых пропорциональна произведению нефиксированного числа единичных векторов.
\end{abstract}

Ключевые слова: тензорные соотношения, тензорные полиномы Лежандра, теорема Гегенбауэра, псевдориманова геометрия.

\section{1. ВВЕДЕНИЕ}

При решении различных задач теоретической и математической физики часто используются достаточно сложные тензорные выражения. Поэтому установление алгебраических и интегральных соотношений для тензоров является актуальной задачей, позволяющей значительно упрощать проведение аналитических вычислений.

Ряд полезных теорем и соотношений, относящихся к тензорной алгебре, можно найти в работах [1]-[4]. Эти результаты представляют несомненный интерес при решении задач нелинейной электродинамики и гравитации. Целью настоящей работы является доказательство теоремы об интегрировании сложного тензорного соотношения с функцией источника для неоднородного уравнения Гельмгольца.

Дадим необходимые определения. Рассмотрим две точки $\mathbf{r}$ и $\mathbf{r}^{\prime}$ трехмерного евклидова пространства. Построим два единичных вектора $\mathbf{M}(\theta, \varphi)=\mathbf{r} / r$ и $\mathbf{N}\left(\theta^{\prime}, \varphi^{\prime}\right)=$ $\mathbf{r}^{\prime} / r^{\prime}$, которые в прямоугольной декартовой системе координат имеют компоненты

$$
\begin{aligned}
M_{x} & =\sin \theta \cos \varphi, & M_{y}=\sin \theta \sin \varphi, & M_{z}=\cos \theta, \\
N_{x} & =\sin \theta^{\prime} \cos \varphi^{\prime}, & N_{y}=\sin \theta^{\prime} \sin \varphi^{\prime}, & N_{z}=\cos \theta^{\prime},
\end{aligned}
$$

${ }^{*}$ Московский государственный университет им. М. В. Ломоносова, Москва, Россия. E-mail: polina@genphys.phys.msu.ru,denisov@srd.sinp.msu.ru 
где $\theta, \varphi$ и $\theta^{\prime}, \varphi^{\prime}$ - сферические углы, определяющие ориентацию векторов $\mathbf{M}$ и $\mathbf{N}$ соответственно.

Используя вектор М, построим бесконечную совокупность тензоров, определяемых в декартовых координатах для четного и нечетного индекса следующими конечными рядами:

$$
\begin{aligned}
& P_{2 k}^{\left(\alpha_{1} \alpha_{2} \ldots \alpha_{2 k}\right)}(\theta, \varphi)=\frac{(-1)^{k}}{2^{2 k}}\left\{\frac{(2 k) !}{(k !)^{2}} \delta^{\left(\alpha_{1} \alpha_{2}\right.} \ldots \delta^{\left.\alpha_{2 k-1} \alpha_{2 k}\right)}+\right. \\
& \left.\quad+\sum_{n=1}^{k} \frac{(-1)^{n}(2 k+2 n) !}{(k+n) !(k-n) !(2 n) !} M^{\left(\alpha_{1}\right.} M^{\alpha_{2}} \ldots M^{\alpha_{2 n}} \delta^{\alpha_{2 n+1} \alpha_{2 n+2}} \ldots \delta^{\left.\alpha_{2 k-1} \alpha_{2 k}\right)}\right\}, \\
& P_{2 k+1}^{\left(\alpha_{1} \alpha_{2} \ldots \alpha_{2 k+1}\right)}(\theta, \varphi)=\frac{(-1)^{k}}{2^{2 k+1}} \sum_{n=0}^{k} \frac{(-1)^{n}(2 k+2 n+2) !}{(k+n+1) !(k-n) !(2 n+1) !} \times \\
& \quad \times M^{\left(\alpha_{1}\right.} M^{\alpha_{2}} \ldots M^{\alpha_{2 n+1}} \delta^{\alpha_{2 n+2} \alpha_{2 n+3}} \ldots \delta^{\left.\alpha_{2 k} \alpha_{2 k+1}\right)}, \quad k>0 .
\end{aligned}
$$

где $\delta^{\alpha \beta}=\delta_{\alpha \beta}$ - тензор Кронекера, и по индексам, заключенным в круглые скобки, производится симметризация:

$$
A^{\left(\alpha_{1} \alpha_{2} \ldots \alpha_{n}\right)}=\frac{1}{N !}(\underbrace{A^{\alpha_{1} \alpha_{2} \ldots \alpha_{N}}+A^{\alpha_{2} \alpha_{1} \alpha_{3} \ldots \alpha_{N}}+\cdots+A^{\alpha_{1} \alpha_{2} \ldots \alpha_{N} \alpha_{N-1}}}_{N !}) .
$$

Совершенно аналогично введем совокупность тензоров, зависящую от угловых координат точки $\mathbf{r}^{\prime}$, для четного и нечетного индекса:

$$
\begin{aligned}
& P_{2 k}^{\left(\alpha_{1} \alpha_{2} \ldots \alpha_{2 k}\right)}\left(\theta^{\prime}, \varphi^{\prime}\right)=\frac{(-1)^{k}}{2^{2 k}}\left\{\frac{(2 k) !}{(k !)^{2}} \delta^{\left(\alpha_{1} \alpha_{2}\right.} \ldots \delta^{\left.\alpha_{2 k-1} \alpha_{2 k}\right)}+\right. \\
& \left.\quad+\sum_{n=1}^{k} \frac{(-1)^{n}(2 k+2 n) !}{(k+n) !(k-n) !(2 n) !} N^{\left(\alpha_{1}\right.} N^{\alpha_{2}} \ldots N^{\alpha_{2 n}} \delta^{\alpha_{2 n+1} \alpha_{2 n+2}} \ldots \delta^{\left.\alpha_{2 k-1} \alpha_{2 k}\right)}\right\}, \\
& P_{2 k+1}^{\left(\alpha_{1} \alpha_{2} \ldots \alpha_{2 k+1}\right)}\left(\theta^{\prime}, \varphi^{\prime}\right)=\frac{(-1)^{k}}{2^{2 k+1}} \sum_{n=0}^{k} \frac{(-1)^{n}(2 k+2 n+2) !}{(k+n+1) !(k-n) !(2 n+1) !} \times \\
& \quad \times N^{\left(\alpha_{1}\right.} N^{\alpha_{2}} \ldots N^{\alpha_{2 n+1}} \delta^{\alpha_{2 n+2} \alpha_{2 n+3}} \ldots \delta^{\left.\alpha_{2 k} \alpha_{2 k+1}\right)}, \quad k>0 .
\end{aligned}
$$

Несложно убедиться, что если всем тензорным индексам в выражениях (1.3) присвоить значение, равное трем, то $P_{2 k}^{\left(\alpha_{1} \alpha_{2} \ldots \alpha_{2 k}\right)}$ перейдет в $P_{2 k}\left(\cos \theta^{\prime}\right)$, а $P_{2 k+1}^{\left(\alpha_{1} \alpha_{2} \ldots \alpha_{2 k+1}\right)}-$ в $P_{2 k+1}\left(\cos \theta^{\prime}\right)$, где $P_{n}(\cdot)$ - полином Лежандра порядка $n$. Поэтому в дальнейшем тензоры $P_{2 k}^{\left(\alpha_{1} \alpha_{2} \ldots \alpha_{2 k}\right)}$ и $P_{2 k+1}^{\left(\alpha_{1} \alpha_{2} \ldots \alpha_{2 k+1}\right)}$ мы будем называть тензорными полиномами.

\section{2. ПОСТАНОВКА ЗАДАЧИ}

При решении различных задач теоретической и математической физики в областях со сферическими границами часто возникает необходимость вычисления интеграла вида

$$
\Psi^{\alpha_{1} \alpha_{2} \ldots \alpha_{k}}(\mathbf{r})=\frac{1}{c} \int \frac{d V^{\prime}}{\left|\mathbf{r}-\mathbf{r}^{\prime}\right|} B^{\alpha_{1} \alpha_{2} \ldots \alpha_{k}}\left(\mathbf{r}^{\prime}\right) \exp \left\{i \Omega\left(t-\frac{\left|\mathbf{r}-\mathbf{r}^{\prime}\right|}{c}\right)\right\},
$$


который является запаздывающим решением тензорного уравнения Гельмгольца

$$
\Delta \Psi^{\alpha_{1} \alpha_{2} \ldots \alpha_{k}}-\frac{1}{c^{2}} \frac{\partial^{2} \Psi^{\alpha_{1} \alpha_{2} \ldots \alpha_{k}}}{\partial t^{2}}=-\frac{4 \pi}{c} B^{\alpha_{1} \alpha_{2} \ldots \alpha_{k}}(\mathbf{r}) e^{i \Omega t},
$$

где трехмерный тензор $B^{\alpha_{1} \alpha_{2} \ldots \alpha_{k}}(\mathbf{r})$ имеет вид

$$
B^{\alpha_{1} \alpha_{2} \ldots \alpha_{k}}(\mathbf{r})=N^{\alpha_{1}} N^{\alpha_{2}} \ldots N^{\alpha_{k}} F\left(r^{\prime}\right)
$$

а $N^{\alpha}=N^{\alpha}\left(\theta^{\prime}, \varphi^{\prime}\right)=\left\{\sin \theta^{\prime} \cos \varphi^{\prime}, \sin \theta^{\prime} \sin \varphi^{\prime}, \cos \theta^{\prime}\right\}$ - трехмерные компоненты единичного вектора. Такие интегралы встречаются, например, в задачах о нелинейно-электродинамической генерации гармоник вращающейся нейтронной звездой [5], в задачах об излучении гравитационных волн электромагнитными волнами в астрофизических условиях и многих других.

При вычислении таких интегралов удобно воспользоваться теоремой сложения Гегенбауэра [6] и представить функцию Грина $e^{-i k\left|\mathbf{r}-\mathbf{r}^{\prime}\right|} /\left|\mathbf{r}-\mathbf{r}^{\prime}\right|$ в виде бесконечного ряда по функциям Бесселя. Известно [6], что

$$
\frac{e^{-i K\left|\mathbf{r}-\mathbf{r}^{\prime}\right|}}{\left|\mathbf{r}-\mathbf{r}^{\prime}\right|}= \begin{cases}-\frac{\pi i}{2 \sqrt{r r^{\prime}}} \sum_{n=0}^{\infty}(2 n+1) J_{n+1 / 2}\left(K r^{\prime}\right) H_{n+1 / 2}^{(2)}(K r) P_{n}(\cos \gamma), & r>r^{\prime}, \\ -\frac{\pi i}{2 \sqrt{r r^{\prime}}} \sum_{n=0}^{\infty}(2 n+1) J_{n+1 / 2}(K r) H_{n+1 / 2}^{(2)}\left(K r^{\prime}\right) P_{n}(\cos \gamma), & r<r^{\prime},\end{cases}
$$

где

$$
\cos \gamma=\frac{\left(\mathbf{r} \mathbf{r}^{\prime}\right)}{|\mathbf{r}|\left|\mathbf{r}^{\prime}\right|}=\cos \theta \cos \theta^{\prime}+\sin \theta \sin \theta^{\prime} \cos \left(\varphi^{\prime}-\varphi\right) .
$$

В результате задача сводится к вычислению интеграла

$$
I^{\alpha_{1} \alpha_{2} \ldots \alpha_{R}}(\theta, \varphi)=\int_{0}^{2 \pi} d \varphi^{\prime} \int_{0}^{\pi} \sin \theta^{\prime} d \theta^{\prime} P_{Q}(\cos \gamma) N^{\alpha_{1}} N^{\alpha_{2}} \ldots N^{\alpha_{R}}
$$

где $Q$ - целое число.

Далее мы докажем несколько утверждений, позволяющих вычислять интегралы вида (2.2) при произвольном числе $R$ тензорных индексов.

\section{3. ДОКАЗАТЕЛЬСТВО ЛЕММ}

Начнем с доказательства двух лемм.

Лемма 1. При $k>0$ тензори $N^{\alpha_{1}} N^{\alpha_{2}} \ldots N^{\alpha_{2 k}} u N^{\alpha_{1}} N^{\alpha_{2}} \ldots N^{\alpha_{2 k+1}}$ могут бъть разложены единственным образом по системе тензоров (1.3):

$$
\begin{aligned}
N^{\alpha_{1}} N^{\alpha_{2}} \ldots N^{\alpha_{2 k}}= & \frac{1}{2 k+1} \delta^{\left(\alpha_{1} \alpha_{2}\right.} \delta^{\alpha_{3} \alpha_{4}} \ldots \delta^{\left.\alpha_{2 k-1} \alpha_{2 k}\right)}+ \\
& +\sum_{m=1}^{k}(4 m+1) \frac{2 k(2 k-2) \ldots(2 k-2 m+2)}{(2 k+1)(2 k+3) \ldots(2 k+2 m+1)} \times \\
& \times P_{2 m}^{\left(\alpha_{1} \alpha_{2} \ldots \alpha_{2 m}\right.} \delta^{\alpha_{2 m+1} \alpha_{2 m+2} \ldots \delta^{\left.\alpha_{2 k-1} \alpha_{2 k}\right)},} \\
N^{\alpha_{1}} N^{\alpha_{2}} \ldots N^{\alpha_{2 k+1}}= & \frac{3}{2 k+3} \delta^{\left(\alpha_{1} \alpha_{2}\right.} \delta^{\alpha_{3} \alpha_{4}} \ldots \delta^{\alpha_{2 k-1} \alpha_{2 k}} P_{1}^{\left.\alpha_{2 k+1}\right)}+ \\
& +\sum_{m=1}^{k}(4 m+3) \frac{2 k(2 k-2) \ldots(2 k-2 m+2)}{(2 k+3)(2 k+5) \ldots(2 k+2 m+3)} \times \\
& \times P_{2 m+1}^{\left(\alpha_{1} \alpha_{2} \ldots \alpha_{2 m+1}\right.} \delta^{\alpha_{2 m+2} \alpha_{2 m+3}} \ldots \delta^{\left.\alpha_{2 k} \alpha_{2 k+1}\right)} .
\end{aligned}
$$


ДоказАтельство. Рассмотрим сначала тензор $N^{\alpha_{1}} N^{\alpha_{2}} \ldots N^{\alpha_{2 k}}$. Этот тензор можно представить в виде суммы многочленов (1.3) и универсального тензора тензора Кронекера с последующей симметризацией по всем тензорным индексам:

$$
\begin{aligned}
N^{\alpha_{1}} N^{\alpha_{2}} & \ldots N^{\alpha_{2 k}}=f_{0} \delta^{\left(\alpha_{1} \alpha_{2}\right.} \delta^{\alpha_{3} \alpha_{4}} \ldots \delta^{\left.\alpha_{2 k-1} \alpha_{2 k}\right)}+ \\
& +\sum_{m=1}^{k} f_{m} P_{2 m}^{\left(\alpha_{1} \alpha_{2} \ldots \alpha_{2 m}\right.} \delta^{\alpha_{2 m+1} \alpha_{2 m+2}} \ldots \delta^{\left.\alpha_{2 k-1} \alpha_{2 k}\right)}
\end{aligned}
$$

где коэффициенты $f_{0}$ и $f_{m}$ не зависят от тензорных индексов $\alpha_{1}, \alpha_{2}, \ldots, \alpha_{2 k}$. В декартовых координатах левые и правые части соотношения (3.2) являются трехмерными тензорами, вид которых не зависит от ориентации трехмерного координатного базиса. Коэффициенты ряда с конечным числом членов, стоящего в правой части этого соотношения, являются скалярами относительно преобразований поворота координатного базиса и не зависят от выбора значений тензорных индексов, т.е. остаются одинаковыми для всех возможных наборов этих индексов. Поэтому если соотношение (3.2) справедливо при каком-то одном наборе значений тензорных индексов, то оно должно быть справедливым и при любом другом наборе значений тензорных индексов.

Коэффициенты ряда в (3.2) можно найти, если рассмотреть соотношение (3.1) при $\alpha_{1}=\alpha_{2}=\cdots=\alpha_{2 k}=3$. В этом случае оно принимает вид

$$
\left(\cos \theta^{\prime}\right)^{2 k}=f_{0}+\sum_{m=1}^{k} f_{m} P_{2 m}\left(\cos \theta^{\prime}\right)
$$

Используя известное разложение [6]

$$
\begin{aligned}
\left(\cos \theta^{\prime}\right)^{2 k}= & \frac{1}{2 k+1} P_{0}\left(\cos \theta^{\prime}\right)+ \\
& +\sum_{m=1}^{k}(4 m+1) \frac{2 k(2 k-2) \ldots(2 k-2 m+2)}{(2 k+1)(2 k+3) \ldots(2 k+2 m+1)} P_{2 m}\left(\cos \theta^{\prime}\right)
\end{aligned}
$$

и учитывая единственность разложения функций по полиномам Лежандра, получим

$$
f_{0}=\frac{1}{2 k+1}, \quad f_{m}=(4 m+1) \frac{2 k(2 k-2) \ldots(2 k-2 m+2)}{(2 k+1)(2 k+3) \ldots(2 k+2 m+1)}, m=1,2, \ldots, k .
$$

Подставляя эти значения коэффициентов в (3.2), приходим к первому из соотношений (3.1).

Докажем теперь, что разложение (3.2) является единственным. Доказательство проведем методом от противного. Предположим, что разложение (3.2) не единственно, т.е. существует еще одно представление

$$
\begin{aligned}
& N^{\alpha_{1}} N^{\alpha_{2}} \ldots N^{\alpha_{2 k}}=g_{0} \delta^{\left(\alpha_{1} \alpha_{2}\right.} \delta^{\alpha_{3} \alpha_{4}} \ldots \delta^{\left.\alpha_{2 k-1} \alpha_{2 k}\right)}+ \\
& \quad+\sum_{m=1}^{k} g_{m} P_{2 m}^{\left(\alpha_{1} \alpha_{2} \ldots \alpha_{2 m}\right.} \delta^{\alpha_{2 m+1} \alpha_{2 m+2}} \ldots \delta^{\left.\alpha_{2 k-1} \alpha_{2 k}\right)}
\end{aligned}
$$


где коэффициенты $g_{0}$ и $g_{m}$ также не зависят от тензорных индексов $\alpha_{1}, \alpha_{2}, \ldots, \alpha_{2 k}$ и отличаются от коэффициентов $f_{0}$ и $f_{m}, m=1,2, \ldots, k$. Тогда, вычитая из $(3.2)$ выражение (3.3), будем иметь

$$
\begin{aligned}
0= & {\left[\frac{1}{2 k+1}-g_{0}\right] \delta^{\left(\alpha_{1} \alpha_{2}\right.} \delta^{\alpha_{3} \alpha_{4}} \ldots \delta^{\left.\alpha_{2 k-1} \alpha_{2 k}\right)}+} \\
& +\sum_{m=1}^{k}\left[(4 m+1) \frac{2 k(2 k-2) \ldots(2 k-2 m+2)}{(2 k+1)(2 k+3) \ldots(2 k+2 m+1)}-g_{m}\right] \times \\
& \times P_{2 m}^{\left(\alpha_{1} \alpha_{2} \ldots \alpha_{2 m}\right.} \delta^{\alpha_{2 m+1} \alpha_{2 m+2}} \ldots \delta^{\left.\alpha_{2 k-1} \alpha_{2 k}\right)} .
\end{aligned}
$$

Это тензорное соотношение должно быть справедливым при любых значениях тензорных индексов, в том числе и при $\alpha_{1}=\alpha_{2}=\cdots=\alpha_{2 k}=3$. В этом случае соотношение (3.4) принимает вид разложения нуля по полиномам Лежандра:

$$
\begin{aligned}
0= & {\left[\frac{1}{2 k+1}-g_{0}\right] P_{0}\left(\cos \theta^{\prime}\right)+} \\
& +\sum_{m=1}^{k}\left[(4 m+1) \frac{2 k(2 k-2) \ldots(2 k-2 m+2)}{(2 k+1)(2 k+3) \ldots(2 k+2 m+1)}-g_{m}\right] P_{2 m}\left(\cos \theta^{\prime}\right) .
\end{aligned}
$$

В силу единственности разложения функций по полиномам Лежандра [7] коэффициенты, стоящие в квадратных скобках в данном равенстве, должны быть равны нулю. Отсюда следует, что разложение (3.2) тензорной функции $N^{\alpha_{1}} N^{\alpha_{2}} \ldots N^{\alpha_{2 k}}$ по тензорным полиномам является единственным.

Совершенно аналогично, представляя тензор $N^{\alpha_{1}} N^{\alpha_{2}} \ldots N^{\alpha_{2 k+1}}$ в виде

$$
\begin{aligned}
N^{\alpha_{1}} N^{\alpha_{2}} \ldots N^{\alpha_{2 k+1}}= & f_{1} P_{1}^{\left(\alpha_{1}\right.} \delta^{\alpha_{2} \alpha_{3}} \ldots \delta^{\left.\alpha_{2 k} \alpha_{2 k+1}\right)}+ \\
& +\sum_{m=1}^{k} f_{m} P_{2 m+1}^{\left(\alpha_{1} \alpha_{2} \ldots \alpha_{2 m+1}\right.} \delta^{\alpha_{2 m+2} \alpha_{2 m+3}} \ldots \delta^{\left.\alpha_{2 k} \alpha_{2 k+1}\right)}
\end{aligned}
$$

и используя известное разложение

$$
\begin{aligned}
\left(\cos \theta^{\prime}\right)^{2 k+1}= & \frac{3}{2 k+3} P_{1}\left(\cos \theta^{\prime}\right)+ \\
& +\sum_{m=1}^{k}(4 m+3) \frac{2 k(2 k-2) \ldots(2 k-2 m+2)}{(2 k+3)(2 k+5) \ldots(2 k+2 m+3)} P_{2 m+1}\left(\cos \theta^{\prime}\right),
\end{aligned}
$$

можно доказать и второе из разложений (3.1). Лемма доказана.

Рассмотрим теперь интегралы вида

$$
J^{\left(\alpha_{1} \alpha_{2} \ldots \alpha_{R}\right)}(\theta, \varphi)=\int_{0}^{2 \pi} d \varphi^{\prime} \int_{0}^{\pi} \sin \theta^{\prime} d \theta^{\prime} P_{Q}(\cos \gamma) P_{R}^{\left(\alpha_{1} \alpha_{2} \ldots \alpha_{R}\right)}\left(\theta^{\prime}, \varphi^{\prime}\right)
$$


где тензорные полиномы $P_{R}^{\left(\alpha_{1} \alpha_{2} \ldots \alpha_{R}\right)}\left(\theta^{\prime} \varphi^{\prime}\right)$ определены выражениями (1.3). Осуществить интегрирование в правой части этого выражения позволяет

Лемма 2. В декартовъх координатах интеграл (3.6) по сфере единичного радиуса имеет вид

$$
\begin{aligned}
J^{\left(\alpha_{1} \alpha_{2} \ldots \alpha_{R}\right)}(\theta, \varphi) & =\int_{0}^{2 \pi} d \varphi^{\prime} \int_{0}^{\pi} \sin \theta^{\prime} d \theta^{\prime} P_{Q}(\cos \gamma) P_{R}^{\left(\alpha_{1} \alpha_{2} \ldots \alpha_{R}\right)}\left(\theta^{\prime}, \varphi^{\prime}\right)= \\
& =\frac{4 \pi \delta_{Q, R}}{(2 R+1)} P_{R}^{\left(\alpha_{1} \alpha_{2} \ldots \alpha_{R}\right)}(\theta, \varphi),
\end{aligned}
$$

где тензорные полиномы $P_{R}^{\left(\alpha_{1} \alpha_{2} \ldots \alpha_{R}\right)}(\theta, \varphi)$ определены выражениями (1.2).

ДоказАтельство. Рассмотрим сначала случай $R=2 k$. В декартовых координатах результатом интегрирования должен быть трехмерный тензор $2 k$-го ранга, симметричный по своим тензорным индексам. В силу определения (2.1) для $\cos \gamma$ он должен зависеть только от углов $\theta$ и $\varphi$. Поэтому тензор $J^{\left(\alpha_{1} \alpha_{2} \ldots \alpha_{R}\right)}(\theta, \varphi)$ может быть записан через произведение единичных векторов $M^{\alpha}$ и тензор Кронекера [8]. Но это произведение можно разложить в ряд с конечным числом членов по тензорным полиномам $P_{2 i}^{\alpha_{1} \alpha_{2} \ldots \alpha_{2 i}}(\theta, \varphi)$ с коэффициентами, не зависящими от тензорных индексов:

$$
\begin{aligned}
J^{\left(\alpha_{1} \alpha_{2} \ldots \alpha_{2 k}\right)}(\theta, \varphi)= & h_{0} \delta^{\left(\alpha_{1} \alpha_{2}\right.} \delta^{\alpha_{3} \alpha_{4}} \ldots \delta^{\left.\alpha_{2 k-1} \alpha_{2 k}\right)}+ \\
& +\sum_{m=1}^{k} h_{m} P_{2 m}^{\left(\alpha_{1} \alpha_{2} \ldots \alpha_{2 m}\right.}(\theta, \varphi) \delta^{\alpha_{2 m+1} \alpha_{2 m+2}} \ldots \delta^{\left.\alpha_{2 k-1} \alpha_{2 k}\right)} .
\end{aligned}
$$

Для определения неизвестных коэффициентов $h_{0}$ и $h_{m}$ возьмем один из простейших наборов тензорных индексов: $\alpha_{1}=\alpha_{2}=\cdots=\alpha_{2 k}=3$. Тогда, с одной стороны, используя определение интеграла $J^{\left(\alpha_{1} \alpha_{2} \ldots \alpha_{2 k}\right)}(\theta, \varphi)$, мы можем записать выражение (3.8) в виде

$$
J^{33 \ldots 3}(\theta)=h_{0} P_{0}(\theta)+\sum_{m=1}^{k} h_{m} P_{2 m}(\theta) ;
$$

с другой стороны, согласно формуле (3.7)

$$
J^{33 \ldots 3}(\theta)=\int_{0}^{2 \pi} d \varphi^{\prime} \int_{0}^{\pi} \sin \theta^{\prime} d \theta^{\prime} P_{Q}(\cos \gamma) P_{2 k}\left(\theta^{\prime}\right) .
$$

Используя известное соотношение [6]

$$
\begin{aligned}
P_{2 k}(\cos \gamma)= & P_{2 k}(\cos \theta) P_{2 k}\left(\cos \theta^{\prime}\right)+ \\
& +2 \sum_{m=1}^{2 k} \frac{(2 k-m) !}{(2 k+m) !} P_{2 k}^{m}(\cos \theta) P_{2 k}^{m}\left(\cos \theta^{\prime}\right) \cos \left(m\left(\varphi^{\prime}-\varphi\right)\right),
\end{aligned}
$$

получим

$$
J^{33 \cdots 3}(\theta)=\frac{4 \pi}{4 k+1} \delta_{Q, 2 k}
$$

Сравнивая выражения (3.7) и (3.10), убеждаемся в справедливости соотношения (3.7) при $R=2 k$.

Совершенно аналогично можно убедиться, что выражение (3.7) справедливо и при $R=2 k+1$. Лемма доказана. 


\section{4. ДОКАЗАТЕЛЬСТВО ИНТЕГРАЛЬНОГО ТЕНЗОРНОГО СООТНОШЕНИЯ}

Докажем следующую теорему.

Теорема. Результат интегрирования произведения тензоров $N^{\alpha_{1}} \ldots N^{\alpha_{2 k}} u$ $N^{\alpha_{1}} \ldots N^{\alpha_{2 k+1}}$ с весовой функиией $P_{Q}(\cos \gamma)$ по поверхности сферы единичного радиуса в декартовой системе координат имеет вид

$$
\begin{aligned}
& I^{\left(\alpha_{1} \alpha_{2} \ldots \alpha_{2 k}\right)}(\theta, \varphi)=\int_{0}^{2 \pi} d \varphi^{\prime} \int_{0}^{\pi} \sin \theta^{\prime} d \theta^{\prime} P_{Q}(\cos \gamma) N^{\alpha_{1}} N^{\alpha_{2}} \ldots N^{\alpha_{2 k}}= \\
&=4 \pi\left\{\frac{\delta_{0 Q}}{2 k+1} \delta^{\left(\alpha_{1} \alpha_{2}\right.} \delta^{\alpha_{3} \alpha_{4}} \ldots \delta^{\left.\alpha_{2 k-1} \alpha_{2 k}\right)}+\right. \\
& \quad+\sum_{m=1}^{k} \frac{2 k(2 k-2) \ldots(2 k-2 m+2)}{(2 k+1)(2 k+3) \ldots(2 k+2 m+1)} \delta_{2 m, Q} \times \\
&\left.\quad \times P_{2 m}^{\left(\alpha_{1} \alpha_{2} \ldots \alpha_{2 m}\right.}(\theta, \varphi) \delta^{\alpha_{2 m+1} \alpha_{2 m+2}} \ldots \delta^{\left.\alpha_{2 k-1} \alpha_{2 k}\right)}\right\} \\
& I^{\left(\alpha_{1} \alpha_{2} \ldots \alpha_{2 k+1}\right)}(\theta, \varphi)=\int_{0}^{2 \pi} d \varphi^{\prime} \int_{0}^{\pi} \sin \theta^{\prime} d \theta^{\prime} P_{Q}(\cos \gamma) N^{\alpha_{1}} N^{\alpha_{2}} \ldots N^{\alpha_{2 k+1}}= \\
&=4 \pi\left\{\frac{\delta_{1 Q}}{2 k+3} \delta^{\left(\alpha_{1} \alpha_{2}\right.} \delta^{\alpha_{3} \alpha_{4}} \ldots \delta^{\alpha_{2 k-1} \alpha_{2 k}} P_{1}^{\left.\alpha_{2 k+1}\right)}(\theta, \varphi)+\right. \\
& \quad+\sum_{m=1}^{k} \frac{2 k(2 k-2) \ldots(2 k-2 m+2)}{(2 k+3)(2 k+5) \ldots(2 k+2 m+3)} \delta_{2 m+1, Q} \times \\
& \times\left.P_{2 m+1}^{\left(\alpha_{1} \alpha_{2} \ldots \alpha_{2 m+1}\right.}(\theta, \varphi) \delta^{\alpha_{2 m+2} \alpha_{2 m+3}} \ldots \delta^{\left.\alpha_{2 k} \alpha_{2 k+1}\right)}\right\} .
\end{aligned}
$$

ДокАЗАтЕЛЬство. Рассмотрим интеграл (2.2). Пусть целочисленное $R$ четное, $R=2 k$. Используя результаты леммы 1 , разложим тензор $N^{\alpha_{1}} \ldots N^{\alpha_{2 k}}$, стоящий под интегралом в (2.2), в ряд (3.1) по тензорным полиномам. Тогда тензор $I^{\left(\alpha_{1} \alpha_{2} \ldots \alpha_{2 k}\right)}$ примет вид

$$
\begin{aligned}
& I^{\left(\alpha_{1} \alpha_{2} \ldots \alpha_{2 k}\right)}(\theta, \varphi)=\int_{0}^{2 \pi} d \varphi^{\prime} \int_{0}^{\pi} \sin \theta^{\prime} d \theta^{\prime}\left\{\frac{1}{2 k+1} \delta^{\left(\alpha_{1} \alpha_{2}\right.} \delta^{\alpha_{3} \alpha_{4}} \ldots \delta^{\left.\alpha_{2 k-1} \alpha_{2 k}\right)}+\right. \\
& \quad+\sum_{m=1}^{k}(4 m+1) \frac{2 k(2 k-2) \ldots(2 k-2 m+2)}{(2 k+1)(2 k+3) \ldots(2 k+2 m+1)} \times \\
& \left.\quad \times P_{2 m}^{\left(\alpha_{1} \alpha_{2} \ldots \alpha_{2 m}\right.}\left(\theta^{\prime}, \varphi^{\prime}\right) \delta^{\alpha_{2 m+1} \alpha_{2 m+2}} \ldots \delta^{\left.\alpha_{2 k-1} \alpha_{2 k}\right)}\right\} P_{Q}(\cos \gamma) .
\end{aligned}
$$

Далее, используя результаты леммы 2, проведем интегрирование в правой части этого равенства. В результате получим

$$
I^{\left(\alpha_{1} \alpha_{2} \ldots \alpha_{2 k}\right)}(\theta, \varphi)=4 \pi\left\{\frac{\delta_{Q 0}}{2 k+1} \delta^{\left(\alpha_{1} \alpha_{2}\right.} \delta^{\alpha_{3} \alpha_{4}} \ldots \delta^{\left.\alpha_{2 k-1} \alpha_{2 k}\right)}+\right.
$$




$$
\begin{aligned}
& +\sum_{m=1}^{k} \frac{2 k(2 k-2) \ldots(2 k-2 m+2)}{(2 k+1)(2 k+3) \ldots(2 k+2 m+1)} \delta_{Q, 2 m} \times \\
& \left.\times P_{2 m}^{\left(\alpha_{1} \alpha_{2} \ldots \alpha_{2 m}\right.}(\theta, \varphi) \delta^{\alpha_{2 m+1} \alpha_{2 m+2}} \ldots \delta^{\left.\alpha_{2 k-1} \alpha_{2 k}\right)}\right\} .
\end{aligned}
$$

Таким образом, первое из соотношений (4.1) нами доказано.

Рассмотрим теперь случай, когда целое число $R$ в выражении $(2.2)$ нечетное, $R=2 k+1$. Используя вторую из формул (3.1) из леммы 1 , представим тензор $I^{\left(\alpha_{1} \alpha_{2} \ldots \alpha_{2 k+1}\right)}(\theta, \varphi)$ в виде

$$
\begin{aligned}
& I^{\left(\alpha_{1} \alpha_{2} \ldots \alpha_{2 k+1}\right)}(\theta, \varphi)=\int_{0}^{2 \pi} d \varphi^{\prime} \int_{0}^{\pi} \sin \theta^{\prime} d \theta^{\prime}\left\{\frac{3}{2 k+3} \delta^{\left(\alpha_{1} \alpha_{2}\right.} \delta^{\alpha_{3} \alpha_{4}} \ldots \delta^{\alpha_{2 k-1} \alpha_{2 k}} \times\right. \\
& \quad \times P_{1}^{\left.\alpha_{2 k+1}\right)}\left(\theta^{\prime}, \varphi^{\prime}\right)+\sum_{m=1}^{k}(4 m+3) \frac{2 k(2 k-2) \ldots(2 k-2 m+2)}{(2 k+3)(2 k+5) \ldots(2 k+2 m+3)} \times \\
& \left.\quad \times P_{2 m+1}^{\left(\alpha_{1} \alpha_{2} \ldots \alpha_{2 m+1}\right.}\left(\theta^{\prime}, \varphi^{\prime}\right) \delta^{\alpha_{2 m+2} \alpha_{2 m+3}} \ldots \delta^{\left.\alpha_{2 k} \alpha_{2 k+1}\right)}\right\} P_{Q}(\cos \gamma) .
\end{aligned}
$$

Интегрируя этот конечный ряд с помощью соотношения (3.7) из леммы 2, приходим к равенству

$$
\begin{aligned}
& I^{\left(\alpha_{1} \alpha_{2} \ldots \alpha_{2 k+1}\right)}(\theta, \varphi)=4 \pi\left\{\frac{\delta_{Q 1}}{2 k+3} \delta^{\left(\alpha_{1} \alpha_{2}\right.} \delta^{\alpha_{3} \alpha_{4}} \ldots \delta^{\alpha_{2 k-1} \alpha_{2 k}} P_{1}^{\alpha_{2 k+1}}(\theta, \varphi)+\right. \\
& +\sum_{m=1}^{k} \frac{2 k(2 k-2) \ldots(2 k-2 m+2)}{(2 k+3)(2 k+5) \ldots(2 k+2 m+3)} \delta_{Q, 2 m+1} \times \\
& \left.\quad \times P_{2 m+1}^{\left(\alpha_{1} \alpha_{2} \ldots \alpha_{2 m+1}\right.}(\theta, \varphi) \delta^{\alpha_{2 m+2} \alpha_{2 m+3}} \ldots \delta^{\left.\alpha_{2 k} \alpha_{2 k+1}\right)}\right\} .
\end{aligned}
$$

Таким образом, и второе из соотношений (4.1) справедливо. Теорема доказана.

СледСтвиЕ. Явные выражения для тензорных полиномов $P_{2 k}^{\left(\alpha_{1} \alpha_{2} \ldots \alpha_{2 k}\right)} u$ $P_{2 k+1}^{\left(\alpha_{1} \alpha_{2} \ldots \alpha_{2 k+1}\right)}$ при нескольких значениях $k$ таковы:

$$
\begin{aligned}
P_{1}^{\alpha}= & M^{\alpha}, \quad P_{2}^{\alpha \beta}=\frac{1}{2}\left(3 M^{\alpha} M^{\beta}-\delta^{\alpha \beta}\right), \\
P_{3}^{\alpha \beta \mu}= & \frac{1}{2}\left(5 M^{\alpha} M^{\beta} M^{\mu}-M^{\alpha} \delta^{\beta \mu}-M^{\beta} \delta^{\alpha \mu}-M^{\mu} \delta^{\alpha \beta}\right), \\
P_{4}^{\alpha \beta \mu \nu}= & \frac{1}{8}\left\{35 M^{\alpha} M^{\beta} M^{\mu} M^{\nu}+\delta^{\alpha \beta} \delta^{\mu \nu}+\delta^{\alpha \mu} \delta^{\beta \nu}+\delta^{\alpha \nu} \delta^{\beta \mu}-\right. \\
& -5\left(M^{\alpha} M^{\beta} \delta^{\mu \nu}+M^{\alpha} M^{\mu} \delta^{\beta \nu}+M^{\alpha} M^{\nu} \delta^{\beta \mu}+\right. \\
& \left.\left.+M^{\mu} M^{\nu} \delta^{\alpha \beta}+M^{\mu} M^{\beta} \delta^{\alpha \nu}+M^{\nu} M^{\beta} \delta^{\alpha \mu}\right)\right\} .
\end{aligned}
$$

Соотношения (4.1) при $1 \leqslant k \leqslant 4$ принимают вид

$$
\int_{0}^{2 \pi} d \varphi^{\prime} \int_{0}^{\pi} \sin \theta^{\prime} d \theta^{\prime} P_{Q}(\cos \gamma) N^{\alpha_{1}}=\frac{4 \pi}{3} P_{1}^{\alpha_{1}} \delta_{1, Q}
$$




$$
\begin{aligned}
& \int_{0}^{2 \pi} d \varphi^{\prime} \int_{0}^{\pi} \sin \theta^{\prime} d \theta^{\prime} P_{Q}(\cos \gamma) N^{\alpha_{1}} N^{\alpha_{2}}=\frac{4 \pi}{3}\left\{\delta^{\alpha_{1} \alpha_{2}} \delta_{0, Q}+\frac{2}{5} P_{2}^{\left(\alpha_{1} \alpha_{2}\right)} \delta_{2, Q}\right\} \\
& \int_{0}^{2 \pi} d \varphi^{\prime} \int_{0}^{\pi} \sin \theta^{\prime} d \theta^{\prime} P_{Q}(\cos \gamma) N^{\alpha_{1}} N^{\alpha_{2}} N^{\alpha_{3}}= \\
& \quad=\frac{4 \pi}{5}\left\{\delta^{\left(\alpha_{1} \alpha_{2}\right.} P_{1}^{\left.\alpha_{3}\right)} \delta_{1, Q}+\frac{2}{7} P_{3}^{\left(\alpha_{1} \alpha_{2} \alpha_{3}\right)} \delta_{3, Q}\right\} \\
& \int_{0}^{2 \pi} d \varphi^{\prime} \int_{0}^{\pi} \sin \theta^{\prime} d \theta^{\prime} P_{Q}(\cos \gamma) N^{\alpha_{1}} N^{\alpha_{2}} N^{\alpha_{3}} N^{\alpha_{4}}= \\
& \quad=\frac{4 \pi}{5}\left\{\delta^{\left(\alpha_{1} \alpha_{2}\right.} \delta^{\left.\alpha_{3} \alpha_{4}\right)} \delta_{0, Q}+\frac{4}{7} P_{2}^{\left(\alpha_{1} \alpha_{2}\right.} \delta^{\left.\alpha_{3} \alpha_{4}\right)} \delta_{2, Q}+\frac{8}{63} P_{4}^{\left(\alpha_{1} \alpha_{2} \alpha_{3} \alpha_{4}\right)} \delta_{4, Q}\right\}
\end{aligned}
$$

Благодарности. Работа выполнена при частичной финансовой поддержке РФФИ (грант № 09-02-13540-ОФИ-Ц).

\section{Список литературы}

[1] А. Л. Бондарев, ТМФ, 101:2 (1994), 315-319.

[2] I. P. Denisova, M. Dalal, J. Math. Phys., 38:11 (1997), 5820-5832.

[3] I. P. Denisova, B. V. Mehta, Gen. Relativ. Grav., 29:5 (1997), 583-589.

[4] И.П. Денисова, Введение в тензорное исчисление и его приложения, УНЦ ДО, М., 2004.

[5] П. А. Вшивцева, В. И. Денисов, И. П. Денисова, Докл. РАН, 387:2 (2002), 178-180.

[6] И. С. Градштейн, И. М. Рыжик, Таблицы интегралов, сумм, рядов и произведений, Физматлит, М., 1971.

[7] В. С. Владимиров, Уравнения математической физики, Наука, М., 1988.

[8] Л. Д. Ландау, Е. М. Лифшиц, Теоретическая физика, т. II: Теория поля, Физматлит, М., 1988. 